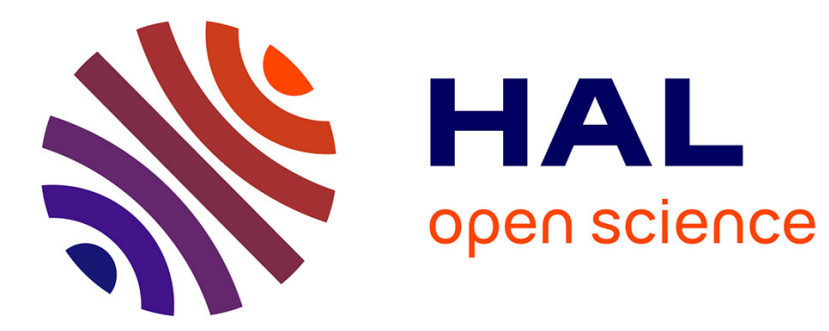

\title{
Terre et échanges chez les Anga (Papouasie Nouvelle-Guinée)
}

Pascale Bonnemère

\section{To cite this version:}

Pascale Bonnemère. Terre et échanges chez les Anga (Papouasie Nouvelle-Guinée). Études rurales, 1992, 127-128, pp.133-158. hal-02176163

\section{HAL Id: hal-02176163 \\ https://hal.science/hal-02176163}

Submitted on 7 Jul 2019

HAL is a multi-disciplinary open access archive for the deposit and dissemination of scientific research documents, whether they are published or not. The documents may come from teaching and research institutions in France or abroad, or from public or private research centers.
L'archive ouverte pluridisciplinaire HAL, est destinée au dépôt et à la diffusion de documents scientifiques de niveau recherche, publiés ou non, émanant des établissements d'enseignement et de recherche français ou étrangers, des laboratoires publics ou privés. 


\section{EHESS}

Terre et échanges chez les Anga (Papouasie Nouvelle-Guinée)

Author(s): Pascale Bonnemère and Pierre Lemonnier

Source: Études rurales, No. 127/128, La terre et le Pacifique (Jul. - Dec., 1992), pp. 133-158

Published by: EHESS

Stable URL: http://www.jstor.org/stable/20125322

Accessed: 08/05/2010 12:32

Your use of the JSTOR archive indicates your acceptance of JSTOR's Terms and Conditions of Use, available at http://www.jstor.org/page/info/about/policies/terms.jsp. JSTOR's Terms and Conditions of Use provides, in part, that unless you have obtained prior permission, you may not download an entire issue of a journal or multiple copies of articles, and you may use content in the JSTOR archive only for your personal, non-commercial use.

Please contact the publisher regarding any further use of this work. Publisher contact information may be obtained at http://www.jstor.org/action/showPublisher?publisherCode=ehess.

Each copy of any part of a JSTOR transmission must contain the same copyright notice that appears on the screen or printed page of such transmission.

JSTOR is a not-for-profit service that helps scholars, researchers, and students discover, use, and build upon a wide range of content in a trusted digital archive. We use information technology and tools to increase productivity and facilitate new forms of scholarship. For more information about JSTOR, please contact support@jstor.org. 


\section{TERRE ET ÉCHANGES CHEZ LES ANGA (PAPOUASIE Nouvelle-GuINíE)}

Dans les hautes terres de NouvelleGuinée, il suffit qu'un pandanus soit coupé, qu'un chasseur étranger ait été aperçu au plus profond d'une forêt, ou qu'une ligne à haute tension s'élève indûment sur le sol d'une tribu pour que l'on passe des cris aux armes et des armes à la guerre. Tout le flou porte sur l'identité du "groupe" qui se mobilise ainsi, car, si comme tant d'autres peuples, les Néo-Guinéens inscrivent leur histoire, leur travail et leurs morts dans la terre, il est généralement impossible de faire coïncider un groupe local et un groupe d'unifiliation ou de préciser ce qui rassemble des personnes qui entreprennent de concert une action politique ou cérémonielle. Du même coup, les modèles d'unifiliation élaborés par les africanistes ne permettent pas de rendre compte de l'organisation politique ou de la tenure foncière dans ces sociétés [Barnes 1962 ; Pouwer 1964 ; Salisbury 1964 ; Scheffler $1985: 2-3]$.

Il y a vingt ou trente ans, l'anthropologie mélanésianiste s'est appliquée à définir la nature du lien social qui rassemble ceux qui se réclament d'une communauté territoriale et agissent en son nom, mais la variété des cas défiait toute généra- lisation ; puis elle est passée à l'étude d'autres traits saillants de ces cultures : les échanges cérémoniels et l'omniprésence de la domination masculine. Sans chercher aucunement à soulever à nouveau la question générale des relations entre groupes de parenté, territoire et communauté d'intérêts, nous essaierons de montrer comment les recherches contemporaines sur les échanges et les théories de la personne affinent notre compréhension du rapport à la terre chez les Anga, dans la cordillère centrale de l'île, en Papouasie Nouvelle-Guinée.

\section{Terre, groupes locaux et parenté : le puzzle néo-guinéen}

En Nouvelle-Guinée, les groupes locaux disposent d'un territoire qui leur est propre et ils peuvent constituer des unités exogames, mais on y trouve souvent une forte proportion d'individus qui, bien que membres du groupe à part entière, ne sont pas des consanguins des premiers habitants du village ou des premiers défricheurs du sol. Il arrive même que les non-agnats l'emportent en nombre, comme chez les Huli des Southern Highlands où les parents en ligne patrilinéaire ne représentent que le quart de la population [Frankel 1986 : 43 ; Glasse 1968 : 29]. Agnats et non-agnats jouissent fréquemment de statuts identiques ; parfois, il n'existe même aucun contexte collectif où l'on puisse les différencier par leur comportement, la distinction se faisant alors entre émigrants récents et membres établis [Scheffler 1985 : 15]. Les cas de double appartenance sont fréquents : il arrive qu'une femme conserve son affiliation clanique lorsqu'elle vit et réside avec un homme d'un autre groupe [ibid. : 11], ou qu'un homme dispose de droits dans plusieurs communautés à la fois, pourvu qu'il participe à leurs activités militaires ainsi 
qu'aux échanges et rituels qui s'y déroulent - cas célèbre, à nouveau, des Huli [Glasse 1968 : 34, 78]. Comme l'a noté H.W. Scheffler [ibid. : 12$15]^{1}$, dans ces sociétés, être l'enfant d'un homme d'un groupe local donné peut être une condition généalogique suffisante pour appartenir à ce groupe, mais ce n'est en rien une condition nécessaire. Là où ils existent, les clans ne forment presque jamais des entités politiques solidaires [La Fontaine 1973 : 38 ; Langness 1964 ; Scheffler 1985 : 15], c'est-à-dire qu'il est rare qu'un clan tout entier participe à une activité - guerre, initiations, échanges cérémoniels - en tant que groupe constitué. Non seulement les individus et même les lignages n'agissent pas ensemble, mais ils s'en gardent bien [Langness 1972 : 927]. Les choses sont d'autant moins simples que nombre de sociétés de Nouvelle-Guinée se caractérisent par l'autonomie des comportements individuels, par la liberté de manœuvre dont dispose l'individu, tant dans le domaine résidentiel qu'en matière d'échanges de richesses, pourtant souvent au centre de la vie collective [Barnes $1962: 7]$. Un jeune homme peut choisir d'aller s'installer dans un autre groupe que celui de son père - au titre de neveu utérin, comme réfugié ou simplement comme ami - et ne décider que fort tard de sa résidence définitive. De même, l'individu dispose de ses propres partenaires dans les échanges cérémoniels, lors desquels ses intérêts sont confrontés à ceux du groupe de parenté ou du groupe local auquel il appartient [Feil 1987: 253 $s q$.]. Et en cas de conflit armé entre deux groupes, il arrive qu'il soit impossible de prédire quel côté un homme appartenant à une tierce partie va aller aider. Si bien que plusieurs auteurs ont vu dans l'individualisme l'élément clef des sociétés qu'ils étudiaient [voir la notion de security circle chez
Lawrence 1984 ; ainsi que Sillitoe $1979: 4-11$ et A.J. Strathern 1979]².

En tout état de cause, il importe de ne pas confondre d'éventuelles institutions patrilinéaires, régissant les rapports entre certains membres d'un village ou d'un hameau, avec les normes et procédés par lesquels un individu se trouve être, ou devient, membre de ce groupe local en tant qu'il constitue une communauté [Glasse 1968 : 138 ; Ryan 1959]. Or, ces normes prennent souvent la forme d'une référence à une idéologie patrilinéaire : alors même qu'ils ne savent pas retracer les liens qui les unissent, entre eux ou à un ancêtre commun, et bien que la profondeur généalogique soit extrêmement faible, il n'est pas rare que les membres de ces groupes se présentent comme des consanguins descendant d'un même "père". Quels sont alors le sens et la fonction d'une telle fiction? Plusieurs auteurs y ont vu un moyen d'affirmer l'unité du groupe [Lepervanche 1967-1968 : 168 ; A.J. Strathern 1969 : 38], notamment en liant la solidarité entre les guerriers à la supériorité masculine [Allen 1984 ; Feil 1987 : 147 ; A.J. Strathern 1969 : 42, 1982a : 37-38]. D'autres, en revanche, se sont attachés à montrer qu'il ne s'agissait pas là de références à une descendance patrilinéaire. Selon H.W. Scheffler [1985 : 13-16], en fait d" "idéologie agnatique", les

1. H.W. Scheffler arrive à la conclusion qu'il n'existe pas de groupes de filiation unilinéaire dans les Highlands.

2. Laissant de côté les débats des années 1960 et 1970 - malheureusement sans toujours clairement démontrer leur éventuelle absence de pertinence, mais non sans ébrécher au passage quelques principes durkheimiens -, plusieurs auteurs ont formé des voux pour que l'on tente d'élaborer "une théorie de l'action sociale plutôt qu'une théorie de la société" [M. Strathern 1988: 96 ; Wagner 1974: 104]. 
groupes de Nouvelle-Guinée ne parlent que de parenté paternelle ou fraternelle et non de filiation : il ne faut pas confondre des obligations entre parents avec des droits et devoirs liés à l'appartenance à un groupe de filiation. Rien ne prouve même que les individus ainsi regroupés se considèrent eux-mêmes comme des agnats [ibid. : 19, note 8] ${ }^{3}$. Ainsi, pour R. Wagner, les "groupes" auxquels on fait allusion dans les Highlands n'existeraient que dans le discours, comme une manière de préciser les catégories virtuelles d'individus qui interviennent dans les échanges de richesses, mais sans que ces personnes réunies pour la circonstance ne constituent jamais des entités sociologiques "délibérément organisées ou idéologiquement régulées" [Wagner 1974 : 110-111]. Bref, l'unifiliation, ou plus généralement la référence à des liens généalogiques, ne sont au mieux qu'un critère de rattachement des individus à un groupe, parmi d'autres. La solidarité des groupes locaux résulte davantage de la communauté de résidence, du travail effectué de concert, de la participation à une même entreprise (guerre, initiations masculines, échanges cérémoniels, rituels divers), ou bien du partage de la nourriture ou des richesses que l'on reçoit ${ }^{4}$.

La résidence dans un village ou hameau - présence d'un individu à un moment donné ou, dans le passé, celle d'un parent - s'accompagne d'un accès aux terres de la communauté locale destinées à la chasse et à l'horticulture. Elle implique également une participation à des activités collectives, considérée par les Néo-Guinéens euxmêmes comme une condition d'appartenance à la communauté [Glasse 1968 : 78 ; A.J. Strathern 1982a : 38]. Mais habiter sur un même territoire revient aussi, souvent, à travailler ensemble [Lepervanche 1967-68: 143] et à se nourrir sur un même sol. A.J. Strathern a montré qu'en absorbant la nourriture qui a poussé sur le sol d'un clan, un enfant ou un immigré acquiert une identité de substance avec les membres du clan en question, identité qui est du même type que celle qu'on obtient en partageant le sang, le sperme, les os, etc. de l'un ou de ses deux parents. La nourriture constitue alors un médiateur entre l'association à un lieu et l'appartenance à un groupe de parenté [A.J. Strathern 1973 : 28-33]. Comme le dit encore J.F. Weiner [1982 : 27], "les gens qui mangent une nourriture qui a poussé sur une même terre sont considérés comme des parents" [cf. également LiPuma 1988 : 100-101].

Cette équation entre nourriture, "nourrissage" et substance (food, nurturance, substance) prend des formes variables dans les Highlands, mais elle se trouve au fondement des échanges entre preneurs et donneurs de femmes, car, par des dons de nourriture ou de richesses aux seconds, les premiers "compensent" la part de substance maternelle que renferme l'enfant qui est incorporé à sa naissance dans le lignage de son père [A.J. Strathern 1981: 211 ; Weiner 1982 : 17-18]. Si l'on ajoute l'idée, souvent explicite, d'un recyclage des principes vitaux lors du retour dans le sol de certaines substances cadavériques $^{5}$, on voit qu'à défaut de présenter une nette équivalence entre filiation et résidence, les NéoGuinéens n'en établissent pas moins des relations

3. Dans le même ordre d'idées, pour M. Strathern [1988: 52], il y aurait lieu de se demander si le souci de solidarité que l'on prête aux Highlanders correspond à une réalité.

4. Cf. J.A. Barnes 1962 : 6 ; P. Brown 1962 ; D.K. Feil 1987 : 125 ; L.L. Langness 1964 ; M. de Lepervanche 1967-68: 143 ; A.J. Strathern $1979: 148$.

5. Cf. par exemple G. Gillison $1983: 38-39 ;$ A. Itéanu $1983: 175$ 177; M. Kahn 1986 : 110 sq. ; A.J. Strathern 1982b: 117-119. 
cruciales entre l'accès à la terre, la parenté et les échanges de nourriture et/ou de richesses qui ponctuent leur vie sociale. Une large part de l'anthropologie mélanésianiste des vingt-cinq dernières années a précisément consisté à tenter de dégager des régularités au sein de ces relations ${ }^{6}$.

\section{Les Anga dans le paysage néo-guinéen}

Descendants directs d'une population parvenue il y a plusieurs millénaires dans la région de Menyamya, les 80000 Anga sont aujourd'hui dispersés sur un peu plus de $15000 \mathrm{~km}^{2}$ de montagnes escarpées, à la jonction de trois provinces de Papouasie Nouvelle-Guinée : Eastern Highlands, Gulf et Morobe $^{7}$. La majorité des tribus ont derrière elles une migration plus ou moins longue, et le vif souvenir des batailles qui ont marqué leur fuite ou leur installation est sans doute pour beaucoup dans leur intérêt pour le fait territorial, car toute terre occupée a été conquise. Même lorsque la forêt est omniprésente et les hommes peu nombreux, il n'est pas un point d'une vallée qui ne soit repéré, approprié, nommé et marqué. Les lignes de crêtes, les torrents, les ruisseaux ou les rochers délimitent le territoire associé à chaque clan, lui-même éventuellement subdivisé à la suite de conflits entre lignages.

Aussitôt franchis la montagne ou le torrent délimitant le territoire de la tribu, on se trouvait dans un monde hostile, terrifiant même, lorsque ses occupants, hommes ou esprits, avaient pour redoutable différence d'être cannibales. Tant les raids contre l'ennemi que les expéditions "commerciales" ne touchaient que les voisins immédiats [Godelier 1969 ; Lemonnier 1981], et l'on garde en mémoire les noms de ceux qui se sont aventurés au-delà. Pour les hommes aujourd'hui âgés de plus de 60 ans, l'in- connu a longtemps commencé bien avant le cercle de montagnes, le défilé ou le méandre qui barrent l'horizon, et ce n'est que sous la sauvegarde d'un officier de patrouille australien qu'ils les ont pour la première fois dépassés. Ignorant tout de l'océan malgré les coquillages dont ils se parent et les monstres marins (pour nous des crocodiles) qui hantent parfois leurs mythes, les Anga n'en étaient pas moins quotidiennement confrontés à une forme d'insularité. On vit et on meurt dans un espace clos, que deux ou trois jours de marche, rarement quatre, permettent de parcourir de part en part.

Alignés sur des crêtes parfois à peine plus larges qu'une maison, ou accrochés aux flancs de profondes vallées, les villages anga se présentaient traditionnellement comme des forteresses végétales, tantôt entourées d'une palissade de planches et d'un infranchissable mur de cannes (Saccharum spontaneum), tantôt enfouies dans le tunnel d'un épais labyrinthe de fins bambous. Car une attaque ennemie était toujours possible et l'état de guerre à peu près permanent : guerre contre quelque groupe menye en expansion, ou contre des réfugiés en quête d'espace, mais aussi, et surtout, combats internes à une tribu, généralement à la suite d'un vol de porc ou d'un adultère. Notons cependant que si la lutte pour la terre était - et demeure ${ }^{8}$ 6. Voir la synthèse présentée par M. Strathern [1984].

7. Lorsqu'aucune source n'est indiquée, les informations comparées concernant les Anga en général ont été recueillies par P. Lemonnier entre 1978 et 1988 ; les données sur les Ankave proviennent des enquêtes de P. Bonnemère dans la vallée de la Suowi (Gulf Province) depuis 1987.

8. Dans un rayon d'un jour de marche autour de Menyamya, on se bat encore régulièrement pour des problèmes de terres. En juin 1990, toute une vallée iqwaye était en émoi car on avait vu passer une petite troupe de 25 Menye parlant d'acheter le droit de s'installer dans une zone à peu près vide d'hommes - mais infestée de malaria-dans les basses terres. 
incontestable autour de Menyamya, dans la majorité des cas la pression démographique n'explique pas la fréquence des luttes armées ${ }^{9}$. La densité de population est vingt ou trente fois moins élevée que chez certains groupes des Western Highlands, et il suffit de survoler l'immense no man's land de forêt qui sépare des groupes ennemis pour se convaincre que, même avec des techniques agricoles moins sophistiquées que celles des groupes évoqués à l'instant - qui pratiquent le billonnage, le compostage et l'engrais animal - il y a toujours amplement d'espace pour cultiver et chasser ${ }^{10}$.

Tous les Anga sont des horticulteurs et, à des degrés divers, des éleveurs de porcs. Le gibier (marsupiaux, porcs sauvages, casoars) est quantitativement marginal dans leur alimentation bien que qualitativement important - car, avec les fruits de quelques arbres de la forêt ${ }^{11}$, il constitue la principale source de protéines -, mais il tient une place fondamentale dans les rituels, notamment au sein des dons entre affins et lors des initiations masculines. La pêche n'est pratiquée régulièrement que dans les groupes situés aux plus basses altitudes (au-dessous de 1000 mètres). Là où le cycle des essarts [Barrau 1972] a été le plus rapide, la forêt tropicale a laissé place à des étendues de savane (Imperata cylindrica et Saccharum spontaneum) plus ou moins importantes, notamment dans les fonds de vallée.

De l'origine commune de ces sociétés résulte une forte homogénéité culturelle, c'est-à-dire que l'on trouve bien davantage de similitudes entre les organisations sociales, les représentations du monde ou les techniques de deux groupes anga quelconques, qu'entre Anga et non-Anga. Cependant, telle qu'on la raconte oralement, et telle qu'on peut la lire dans le paysage, leur histoire est celle d'une longue dispersion à partir de la vallée de Menyamya, aujourd'hui déforestée sur des milliers d'hectares. Ce mouvement s'est accompagné d'un processus de différenciation linguistique : on compte actuellement douze langues anga, dont les locuteurs ne se comprennent pas d'un groupe à l'autre, à de rares exceptions près.

Tous les Anga possèdent des clans patrilinéaires. Certes, là encore, il n'existe pas de correspondance stricte entre un groupe de parenté et une communauté locale. D'abord, il est fréquent que les membres d'un clan donné se répartissent entre plusieurs hameaux qui, eux-mêmes, regroupent des hommes de plus d'un clan ; ensuite, parmi toutes les unités sociales qui agissent en tant que communautés, on trouve souvent des ensembles d'individus partiellement définis par référence à un lieu : c'est en fonction de leur résidence que les membres d'une vallée ou d'un village prennent part à un combat, sont globalement soupçonnés d'une attaque de sorcellerie, ou organisent un repas cérémoniel. Mais il existe aussi divers

9. Comme l'a rappelé P. Sillitoe [1978:269], bien des sociétés de Nouvelle-Guinée se font la guerre sans but de conquêtes territoriales. Dans les années 1960, plusieurs auteurs [Meggitt 1965 ; Rappaport 1968: 27] ont également avancé l'idée que le degré de patrilinéarité et de patrilocalité variait selon la pression sur la terre, hypothèse qui comporte trop de contreexemples pour être retenue [A.J. Strathern 1969 : 39-4I].

10. La densité de population moyenne est de 5 ou $6 \mathrm{hab} . / \mathrm{km}^{2}$, mais elle varie en fait de près de $25 \mathrm{hab} . / \mathrm{km}^{2}$ chez les Kapau à $1 \mathrm{hab} . / \mathrm{km}^{2}$ dans les groupes du sud-ouest. Chez les Ankave. dont il va être longuement question, elle est de l, I hab. $/ \mathrm{km}^{2}$.

11. Le pandanus “à noix” (Pandanus julianetti), le pandanus "rouge" (Pandanus conoideus), l'arbre à pain (Artocarpus altilis), Pangium edule et Terminalia catappa, en proportion variable selon les groupes; on ne les trouve que rarement tous présents sur le territoire d'une tribu. 
contextes dans lesquels les membres d'un clan ont incontestablement des intérêts qui leur sont propres et, en tout premier lieu, lorsque l'exploitation ou la défense d'un territoire commun sont en jeu. Et même si des étrangers interviennent parfois dans les affaires du clan, c'est pour soutenir ces intérêts qui sont ceux d'un seul clan. Dans les groupes où le mariage s'accompagne du versement d'une compensation matrimoniale, la patrilinéarité est également déterminante dans la définition des parents qui aident à rassembler celle-ci ou qui se la partagent.

À l'instar des sociétés des Eastern Highlands, les tribus anga procèdent régulièrement à l'initiation des jeunes garçons. Partout en effet, la guerre, les rapports hommes/femmes et les initiations forment un complexe de croyances et de pratiques qui se renforcent mutuellement et sont au centre de l'organisation sociale, même si la domination masculine prend des formes et une intensité variable selon les groupes. Mais à la différence de ce qui se passe chez leurs voisins du nord, les initiations masculines anga sont totalement disjointes des paiements qui accompagnent le cycle de vie et du versement des compensations pour homicide [Lemonnier 1990]. On ne trouve pas chez les Anga de grands échanges cérémoniels de viande de porc ou d'animaux vivants. Dans les tribus où l'alliance ne prend pas la forme d'un échange de "sœurs" (réelles ou classificatoires), la viande de porc est cependant un important complément du gibier dans les dons que les preneurs de femmes offrent à leurs affins, tant avant qu'après le mariage, mais c'est le gibier qui joue ici un rôle essentiel.

Les Anga se distinguent donc de nombre de sociétés de Nouvelle-Guinée de deux manières : d'une part la majorité d'entre eux ignorent tout des échanges cérémoniels, et ce sont les initiations qui constituent la grande affaire collective ; en corollaire, le pouvoir politique ne fait aucune place aux organisateurs d'échanges "économiques" qui sont une institution centrale des sociétés des Highlands. Bref, ils n'ont ni Big men ni hiérarchies sociales fondées sur la manipulation de richesses. Chez eux, ce sont des "Grands hommes" - guerriers hors pair, maîtres des initiations, chamanes - qui s'élèvent un peu au-dessus des autres membres masculins de la société, jouissant d'un pouvoir aussi limité que les contextes particuliers dans lesquels ils l'exercent [Godelier 1982 : 253-290]. Mais, si en Nouvelle-Guinée ces sociétés sont repérables entre toutes, elles n'en diffèrent pas moins profondément entre elles, et, précisément, sur des points qui alimentent maints débats anthropologiques : les formes du mariage, les rapports hommes-femmes, les théories du corps et l'organisation des initiations masculines variant de manière étonnante à l'intérieur même de la culture anga.

\section{Brousses de chasse et terres cultivables}

Divers produits de la forêt peuvent être collectés par tous les membres d'une tribu, sans distinction de clan ou de lignage : champignons, légumes à feuilles, lianes et bois ; tout juste demande-t-on l'autorisation (qui n'est jamais refusée) de couper les herbes ou les feuilles de pandanus utilisées pour couvrir un toit, ou de prélever l'écorce destinée à un piège à anguilles. Seule exception à ce libre usage des matériaux : les Menye interdisent à autrui de couper du bois de chauffe dans ce qui leur reste de forêt.

L'accès au gibier est beaucoup plus limité. Les membres d'un clan peuvent librement chasser ou 
installer des pièges dans d'immenses étendues de forêt, généralement d'un seul tenant. Pour les autres hommes de la tribu, la règle - identique dans tous les groupes anga - est que l'on peut tuer n'importe quel gibier aperçu depuis un chemin. On peut ainsi capturer à la main un marsupial blotti dans un trou d'arbre, ou tirer à l'arc sur l'oiseau, le porc sauvage ou, fait rarissime, le casoar qui viendrait à croiser votre route. Il est même alors permis de poursuivre l'animal blessé. En revanche, il est tout à fait interdit de s'enfoncer dans la forêt d'autrui et, d'une manière générale, d'y passer la nuit : quiconque est surpris à dormir sur le territoire d'un clan autre que le sien est immédiatement suspecté d'avoir voulu chasser ou piéger indûment, et des combats peuvent s'ensuivre. Ce n'est qu'exceptionnellement, et après y avoir été invité, que l'on peut aller chasser dans la brousse d'autrui.

Mais toutes les ressources du territoire d'un clan donné ne sont pas exploitées collectivement, tant s'en faut. D'abord, nul ne peut s'emparer d'un gibier déjà pris, ni même s'approcher du piège, lorsque sa pose a été signalée, soit oralement, soit par quelque marque disposée sur le sol. Même entre frères, on ne s'avise pas de prendre un tel gibier sans l'apporter immédiatement au piégeur ou sans le prévenir. Ensuite, certains produits de la forêt sont réservés à celui qui, le premier, les a découverts ou entretenus. C'est surtout le cas des pandanus (Pandanus conoideus et Pandanus julianetti) et des palmiers-aréquiers (Areca catechu), sur lesquels on exerce dans tous les groupes anga des droits individuels jalousement défendus. Les ficus dont l'écorce battue fournit les capes et les pagnes sont également l'objet d'un strict droit d'usage dont le non-respect peut entraîner des combats internes ou une guerre intertribale. Plus rarement (Menye, Sambia, Jeghuje, Watchakes), mais avec autant de passion, c'est également de manière individuelle que l'on veille sur les petits monticules de compost dans lesquels incubent des œufs de gallinacées sauvages (Aepypodius arfakianus, un mégapode).

La même référence au premier travail investi dans une partie du territoire se trouve au fondement des normes qui réglementent l'accès aux terres cultivables, qui, rappelons-le, sont - ou ont été un jour - une portion de la forêt du clan. Dans le cas général, un jardin est ouvert dans la forêt à l'endroit où une zone de culture se trouvait déjà 15 à 30 ans auparavant. Les principales plantes cultivées varient suivant l'environnement et la situation du groupe par rapport aux zones de pénétration des colons européens. Le taro (Colocasia esculenta), la patate douce (Ipomoea batatas), le bananier (Musa sapientum) et la canne à sucre (Saccharum officinarum) sont partout présents, mais la patate douce domine aujourd'hui dans la diète des groupes du nord, où les jardins s'étendent entre 1200 et 2200 mètres, tandis que le bananier était le principal cultivar chez les groupes du sud et de l'ouest, situés plus bas. Introduit au tournant du siècle par les porteurs chinois des explorateurs allemands [Barrau 1962 : 105], mais parvenu sur place seulement dans les années 1950, le "taro" (Xanthosoma sagittifolium) est en passe de constituer l'aliment principal chez ces derniers, car le climat lui est favorable.

Les Anga élèvent en semi-liberté des porcs auxquels une partie des produits des jardins est destinée. Les mâles étant castrés, les truies sont couvertes par les verrats sauvages qui rôdent à la limite de la zone cultivée. Les animaux se nourris- 
sent des tubercules (patates douces crues ou taros cuits) que leur préparent les femmes, mais aussi des divers détritus des villages ainsi que de tout ce qu'ils peuvent déterrer au cours de leurs divagations dans les anciens jardins ou dans la forêt. Notons cependant qu'ils sont relativement peu nombreux : avec environ 0,5 animal par habitant ${ }^{12}$, on est très loin des densités rencontrées dans les Western Highlands. Les jardins n'en sont pas moins le plus souvent entourés d'une barrière destinée à empêcher les porcs, domestiques ou sauvages, de saccager les cultures.

Dans un essart, la majorité des arbres sont abattus - naguère encore à l'aide d'outils de pierre [Blackwood 1950 ; Godelier et Garanger 1973] -, mais quelques-uns sont seulement ébranchés, soit pour qu'ils sèchent sur pied afin de constituer du bois de chauffe, soit pour que leurs racines retiennent le sol, car les pentes cultivées dépassent souvent 50 degrés et les Anga ne connaissent pas les terrasses $^{13}$. On peut aussi choisir de laisser des arbres çà et là afin d'abriter les jeunes pousses du soleil pendant quelques semaines. Dans la majorité des groupes, les troncs sont débités sur place pour obtenir les pieux avec lesquels on fabrique les barrières dont on entoure les cultures ; d'un jardin à l'autre, les pieux en bon état sont réutilisés, surtout en zone de savane où il est plus avantageux de transporter des morceaux de barrière que d'aller abattre des arbres au loin. Partout, les hommes coupent les arbres et construisent les éventuelles barrières de jardins, les femmes rejetant les branchages vers la périphérie de l'essart et coupant les broussailles. Ce sont elles également qui plantent et récoltent la majorité - voire la totalité - des espèces, et qui, jour après jour, effectuent le désherbage des parcelles ${ }^{14}$. Mais les hommes prennent soin des pandanus et des palmiersaréquiers, et en récoltent les fruits. Les groupes du sud-ouest ne pratiquent le brûlis qu'à toute petite échelle : au lieu d'enflammer l'ensemble du futur jardin, on ne brûle que quelques petits tas de branchages, notamment là où du tabac sera planté.

Chez tous les Anga, les descendants en ligne agnatique du premier défricheur d'une terre en disposent librement. Dans la pratique, un père répartit l'ensemble de ses terres horticoles entre ses fils ; s'il décède avant d'avoir pu le faire, c'est son fils aîné qui indique aux plus jeunes le secteur où chacun ira désormais cultiver. Comme d'autres l'ont déjà remarqué [Godelier 1969 ; Lory 1982 : 251], il importe ici de distinguer entre les droits du "propriétaire" du lieu (ou de l'ayant droit principal, comme on voudra) et les droits d'usage qui peuvent s'y appliquer. Dans plusieurs groupes du nord, les Baruya et les Watchakes en particulier, en contrepartie de l'aide apportée par un homme lors de l'abattage des arbres ou de la construction de la barrière du jardin, sa sœur, sa mère ou son épouse reçoit une parcelle de terre à cultiver. Ceux qui participent à un tel travail collectif sont des beaux-frères ou des co-initiés, mais une femme

12. Ces chiffres, les seuls disponibles, proviennent de nos observations chez les Baruya et chez les Ankave.

13. Mais ils allongent parfois des troncs sur le sol, perpendiculairement à la ligne de plus grande pente, ce qui constitue une ébauche de terrasse. Rappelons qu'ailleurs dans l'île, notamment chez les Dani d'Irian Jaya, on construit de véritables terrasses, maintenues par des murets en bois [Heider 1970:36 et planche 6].

14. Dans plusieurs groupes (du centre et du nord), seuls les hommes peuvent couper la canne à sucre [Lemonnier 1982 : 243]. Notons que dans certains groupes des Highlands, la participation des hommes à l'agriculture peut être très importante [Sillitoe $1983: 176$ ]. 
quelconque qui, de sa propre initiative, aide à porter des pieux ou à nettoyer le sol, se voit de même attribuer une parcelle. Notons encore que, dans l'un et l'autre groupe, une part non négligeable de cette entraide dans le défrichage intervient notamment lorsqu'il s'agit de produire et de rassembler de la nourriture en vue des initiations masculines. Parfois, la parenté suffit seule à obtenir le droit de cultiver une terre, comme chez les Menye, où l'on peut se voir offrir une parcelle, alors même que l'usager principal du jardin l'a préparée entièrement seul. Dans d'autres groupes enfin, il n'existe aucune forme de travail en commun dans le jardinage : alors que dix ou quinze Baruya, par exemple, préparent un jardin en une quinzaine de jours, un couple ankave ou kapau travaillera absolument seul pendant de longs mois.

Mais, outre la propriété et le droit d'usage d'un sol agricole, il existe un troisième type de relation à la terre, qui consiste à se voir accorder la possibilité de récolter une partie de ce qui pousse sur une parcelle, indépendamment, cette fois, de toute participation à la production. Ceux à qui l'on permet de se ravitailler ainsi sont des parents (frères et sœurs, affins, maternels) ou, moins souvent, des amis (des co-initiés notamment). Ici, on trouve à nouveau chez les Anga toute une gamme de pratiques allant de l'autorisation de se servir librement à l'interdiction la plus absolue de pénétrer dans le jardin d'autrui. Dans certains groupes, le parent en question peut récolter ce dont il a besoin et avertir ensuite de son passage ; dans d'autres, en revanche, on doit demander l'autorisation avant de se rendre dans un jardin, et tout prélèvement révélé après coup est considéré comme un vol ; ailleurs encore, on ne peut se rendre dans un jardin qu'accompagné de l'épouse de celui qui vous a convié.
À côté de ces pratiques concernant l'utilisation plus ou moins directe du jardin d'autrui, existe partout la possibilité de se faire prêter de la terre. Le plus souvent, c'est un beau-frère (frère d'épouse ou mari de sœur, indifféremment) que l'on accueille ainsi sur son sol, pour un temps plus ou moins long; il est même inconcevable de refuser de la terre à un affin qui en réclame ${ }^{15}$. Beaucoup plus rarement, un homme peut demander un accès à la terre de ses parents maternels. Mais, dans tous les cas, on n'obtient ainsi, au moins théoriquement, que des droits d'usage limités, non transmissibles : chez les Anga, une femme dispose du droit de cultiver la terre de son lignage, et elle ne se prive pas de s'y installer avec son mari ou ses enfants, mais ce droit s'éteint à sa mort [Godelier 1982 : 25]. Théoriquement, car, à la longue, il arrive que des affins continuent d'exploiter régulièrement une terre qui ne leur avait été allouée qu'à titre temporaire. Lorsqu'ils résident en permanence à proximité, et si, de surcroît, ils deviennent aussi nombreux que leurs hôtes, la terre qu'ils occupent est un jour considérée comme la leur, au terme de ce qui n'est qu'une pacifique conquête par le canal de l'affinité. Notons que si un homme et ses descendants peuvent ainsi "faire souche" chez des affins, on ne considère jamais pour autant qu'une femme leur a transmis un droit sur la terre, mais qu'ils ont été accueillis par un beau-frère ou un oncle maternel.

Une dernière relation générale entre les Anga et leur terre passe par les morts. Les formes de traitement des cadavres varient selon les groupes,

15. Il n'y a guère qu'autour de Menyamya, où aucune observation directe n'a été effectuée, que cette possibilité de refus est évoquée par les informateurs. 
puisque le corps est tantôt "fumé" pendant plusieurs semaines (Kapau, Menye,Watchakes), tantôt placé rapidement dans une sépulture (Ankave, Baruya, Langimar, Sambia, Yoyue), le plus souvent une plate-forme mortuaire ${ }^{16}$. La localisation de ces plates-formes diffère elle-même d'une tribu à une autre : le plus souvent, on l'érige dans un jardin de la personne décédée, ou à l'intérieur d'un bosquet de bambous, de pandanus ou d'aréquiers, mais les Menye, les Langimar et les Kapau placent aussi leurs morts dans des abris sous roche ou dans des creux de falaises. Quoi qu'il en soit, dans tous les cas, les fluides cadavériques tombent sur un sol potentiellement cultivable. En effet, même là où le cadavre est longuement desséché, les fluides de décomposition s'écoulent sous la maison où il est exposé, c'est-à-dire en définitive dans un enclos domestique cultivé. Bien que l'information sur ce point soit lacunaire, on connaît plusieurs groupes - notamment les Baruya [Godelier 1982 : 73] et les Ankave - qui plantent sous la plate-forme mortuaire diverses boutures qui sont ensuite replantées ailleurs, si bien qu'il est clair que l'idée d'un cycle des substances vitales est incontestablement présente chez les Anga, même si elle ne fait pas l'objet de commentaires particuliers ${ }^{17}$. Dans plusieurs groupes, ni les enfants ni les vieillards n'étaient fumés ou dépecés ${ }^{18}$, comme si seuls les corps adultes contenaient un pouvoir susceptible d'entrer dans la terre, ce qui va dans le même sens.

Quant aux esprits des morts, pour les Baruya, les Sambia et les Watchakes, une partie au moins d'entre eux veille sur les brousses de chasse [Godelier 1982 : 73 ; Herdt $1987: 43$ ]. Les seuls renseignements disponibles pour les Anga du sud concernent les Ankave : là ce sont des esprits de la forêt qui veillent sur le gibier, d'une manière générale, et non sur la brousse d'un clan particulier ; les esprits des morts, eux, ne s'éloignent jamais des hameaux.

\section{Les Ankave : des Anga de la forêt}

Originaires du voisinage immédiat de Menyamya - leurs ancêtres sont sortis de sous la terre par un trou de rocher situé près d'une falaise qui se trouve être aussi le berceau des Baruya et de plusieurs autres groupes -, les Ankave sont des réfugiés. Ils occupent trois étroites vallées sur les contreforts sud-ouest du Krakte Range, à la périphérie de la région anga. Borné vers l'est par un mur de hautes montagnes, leur territoire presque exclusivement couvert de forêt s'ouvre à l'ouest sur les basses terres du golfe de Papouasie, à peu près vides d'hommes. Habitats et cultures s'échelonnent entre 700 et 1400 mètres. Au nombre d'environ 900 , ils présentent une densité de population extrêmement faible, de l'ordre de $1,1 \mathrm{hab} . / \mathrm{km}^{2}$, soit 5 fois moins que la moyenne anga.

Arrivés sur leur actuel territoire à l'issue d'une pérégrination longue de plusieurs siècles, voire

16. Les seules exceptions concernent les Baruya, dont M. Godelier [1982 : 73] signale qu'ils étaient parfois également enterrés, selon leur clan ou leur statut (chamanes).

17. Notons qu'aucune relation n'a pu être dégagée entre les théories locales de la composition de l'individu et les modes de traitement des cadavres: ces théories varient beaucoup selon les groupes, alors que, jusqu'à plus ample analyse, le "recyclage" des substances corporelles prend partout des formes identiques.

18. C'est le cas chez les Ankave et les Baruva (Godelier : communication personnelle); chez les Kapau, les vieilles femmes sont exclues [Blackwood 1978 : 136] ; chez les Jeghuve [Fischer 1968:173-174] et chez les Watchakes, ce sont les bébés qui sont exclus. 
millénaires, ils se sont généralement alliés par le mariage avec les groupes qui s'y trouvaient, pour quelque temps au moins. Souvent aussi, ils ont combattu et repoussé vers l'ouest les groupes les plus faibles, qui se sont littéralement dissous dans le golfe de Papouasie. Or, ce processus, qui est l'ultime étape des mouvements de population autour de Menyamya, s'observe encore aujourd'hui. Année après année, il se trouve toujours 6 ou 8 personnes - une famille, parfois un petit clan qui descendent vers la Papouasie, à la suite de quelque dissension. Dans les basses terres, la malaria endémique tue régulièrement ${ }^{19}$. Tout aussi régulièrement, on soupçonne des attaques de sorcellerie, dont on se venge en tuant les coupables présumés à coups de flèches ou de hache. De plusieurs clans ankave ainsi chassés vers l'ouest, il ne reste plus aujourd'hui que le nom. La plus proche route s'arrête au poste administratif de Menyamya, dans la province de Morobe, et il faut deux à quatre jours de marche pour l'atteindre. Isolés au fin fond de la province du Gulf, l'une des plus pauvres du pays, les Ankave sont ignorés par les autorités de Kerema, le centre administratif dont ils dépendent officiellement. Dans la vallée de la Suowi - celle étudiée ici - des évangélistes luthériens originaires d'autres groupes anga tentent régulièrement de s'implanter, mais ils sont tantôt chassés par les Ankave, tantôt découragés par leur désintérêt pour la bonne parole. Si bien qu'on n'y trouve ni école, ni infirmerie, ni "boutique". Seule la vallée d'Angai, au sud du territoire, dispose aujourd'hui d'une piste d'aviation, d'accès difficile et très peu fréquentée. Deux ou trois dizaines d'hommes sont allés travailler en plantation à partir de la fin des années 1960, mais depuis l'Indépendance (1975), les rares
Ankave qui voyagent ne quittent leur vallée que pour quelques jours, le temps de vendre des capes d'écorce à leurs "amis" iqwaye. Par leurs fables terrifiantes sur les mœurs cannibales des Menye ou la brutalité des gendarmes, les Iqwaye les arrêtent en chemin, histoire de leur vendre au double du prix le kilo de riz ou le poisson en boîte que le commerce des capes leur permet d'acheter.

Globalement, leur organisation sociale est semblable à celle des autres groupes anga : il n'existe pas d'échanges cérémoniels de grande envergure et aucune hiérarchie n'est fondée sur la manipulation de richesses ; les initiations masculines tiennent une place cruciale dans la vie du groupe et dans la reproduction de l'ordre social ; on trouve chez eux des Grands hommes. Elle présente cependant plusieurs originalités, notamment par rapport aux Anga du nord. Le mariage par échange direct de femmes n'existe pas en tant que tel et toutes les alliances s'accompagnent du versement d'une compensation matrimoniale. Ensuite, bien que la domination masculine y soit incontestable, les rapports entre les sexes sont moins brutaux et antagonistes qu'ailleurs ; en particulier, les femmes ne sont pas systématiquement dénigrées et leur rôle, complémentaire de celui des hommes, n'est pas dévalorisé [Bonnemère 1993a : 353, 564].

Bien qu'ils vivent au milieu de la forêt, et malgré une densité de population de chasseurs-cueilleurs, les Ankave sont essentiellement des horticulteurs ("taro", patate douce, bananier). Ils exploitent régulièrement deux arbres de la forêt dont les saisons se complètent, le Pandanus conoideus et le

19. Dans la vallée de la Suowi, où la malaria n'est "que" méso-endémique, le taux de mortalité infantile est de 349\%. Le taux de mortalité global est de $70 \%$. 
Pangium edule ${ }^{20}$, des fruits desquels on tire des sauces consommées avec des tubercules. Comme pour tous les Anga, le gibier a surtout pour eux un rôle cérémoniel : les marsupiaux sont chassés ou piégés pour être offerts aux donneurs de femmes, à une épouse qui vient d'accoucher, ou lors des initiations masculines. Les porcs sont nourris de "taros" cuits ; leur élevage est relativement peu développé mais le nombre d'animaux par habitant $(0,5)$ est dans la moyenne de ce qui se rencontre chez les Anga. Notons enfin que les Ankave sont les seuls Anga à ne pas produire de sel végétal.

Leur manière d'exploiter le territoire se caractérise par une mobilité résidentielle élevée. Outre un enclos domestique et une maison dans un hameau, établi entre 1100 et 1400 mètres d'altitude, chaque famille dispose d'abris dans ses jardins ou dans les zones plus basses où elle se rend pour récolter des fruits (de Pangium edule ou d'Artocarpus altilis), piéger les anguilles ou prélever les écorces de ficus avec lesquelles on fabrique les capes et les pagnes. Au bout du compte, les gens passent la moitié, voire les deux tiers de leur temps, hors du hameau dans lequel ils ont leur résidence principale, même s'ils n'en sont parfois éloignés que d'une demi-heure à peine. À cette mobilité annuelle s'en ajoute une autre qui correspond au cycle des jardins. Lorsqu'une famille ouvre un nouvel essart, tous les deux ou trois ans, il est fréquent qu'elle défriche une portion de son territoire qui se situe à l'opposé du site précédent. Il arrive même qu'un Ankave ouvre un jardin uniquement dans le but d'affirmer son droit d'usage sur une portion de forêt : mal clos, le jardin est abandonné et ruiné par les porcs, aussitôt que défriché. Consciente ou non, la stratégie est patente lorsqu'un même homme possède simultanément quatre jardins (au lieu de deux, habituellement) dans quatre secteurs éloignés d'un territoire que les membres de son clan ont bien du mal à occuper entièrement.

Cette originalité dans le mode d'occupation du territoire se résume spectaculairement dans le paysage : telle vallée qui, chez les Baruya, par exemple, serait couverte d'un entrelacs de jardins et de savane, les crêtes étant seules couvertes de forêt, se présente chez les Ankave comme une étendue presque entièrement boisée dans laquelle un oil peu expérimenté discerne à peine les zones où les cultures ont cessé depuis plus de trois ans. De même, au lieu que de gros villages permanents soient aisément repérables à plusieurs kilomètres, seuls quelques milliers de mètres carrés de savane signalent les petits hameaux ankave, et il faut qu'une fumée s'élève pour que l'on devine un abri dans la forêt.

Chez les Ankave, l'accès aux zones cultivables et aux brousses de chasse suit globalement les mêmes règles que chez les autres Anga. Là encore, et bien que la terre soit particulièrement abondante, il n'est pas un secteur du territoire qui ne soit approprié. Les principaux marqueurs sont ici les pandanus rouges et les palmiers-aréquiers, qui bornent aussi bien les anciens jardins que la forêt profonde, alors que chez les Anga du nord, c'est le Pandanus julianetti qui constitue le marqueur par excellence, celui dont l'exploitation illégitime peut conduire à de graves conflits. Mais, à la différence de ce qui se passe chez les Baruya et les Sambia, pour qui les esprits des ancêtres d'un clan rendent gravement malades ou tuent les chasseurs qui n'appartiennent pas à ce clan, les esprits invisibles qui habitent la forêt ankave ne sont pas ceux des

20. Voir P. Bonnemère 1993b. 
morts, mais des pəjəฤə’ āwə', des êtres qui veillent sur le gibier en général, et non sur un territoire clanique donné. Pourvu qu'ils en demandent l'autorisation, des affins ou des parents maternels peuvent avoir accès au territoire forestier d'autrui, mais pour en prélever certains produits seulement. Ils peuvent s'approvisionner en noix d'arec et en fruits du pandanus rouge, mais ils ne peuvent $y$ chasser.

L'intérêt pour les anguilles est une autre particularité ankave. À l'issue de la cérémonie par laquelle on clôt le deuil en même temps que l'on chasse l'esprit du mort, elles sont données aux parents croisés classificatoires du défunt. De tous les droits "fonciers", ceux relatifs aux portions de torrents sont les plus sévèrement délimités et contrôlés, et plusieurs conflits ayant entraîné mort d'homme avaient pour origine leur non-respect. Chaque frère d'un même père connaît le bief dans lequel lui seul peut poser ses pièges à anguilles, si bien que cet animal est le produit le plus étroitement associé à une partie du territoire lignager.

C'est par l'habituelle référence au premier défricheur d'une terre que l'on justifie un droit d'usage sur celle-ci. Dans une certaine mesure, une femme peut transmettre ce droit à son mari ou à ses enfants, mais les droits acquis en ligne patrilinéaire l'emportent toujours : un Ankave ne peut cultiver la terre d'un affin ou d'un parent maternel qu'avec son accord. Les premiers clans ankave n'en ont pas moins misé sur une politique de mariage avec les groupes qui se trouvaient déjà dans les vallées pour conforter leur installation. Assurément, un beau-frère ou un neveu utérin ne peut rien exiger de ses affins ou de ses maternels, mais dans la pratique et dans le long terme, ce sont de tels liens de parenté qui sont utilisés pour occuper progressive- ment et pacifiquement la terre d'un autre clan dont la démographie est déclinante.

Fait remarquable : que l'on soit provisoirement invité ou que l'on reçoive définitivement une terre, on s'installe à côté de son hôte, et non dans ses jardins. Car, alors que chez les Anga du nord, la coopération dans le travail - entre beaux-frères, en particulier - est constitutive de l'alliance, la coopération est quasi inexistante chez les Ankave. Par exemple, lorsqu'il arrive que deux beauxfrères, ou un beau-père et son gendre, défrichent "ensemble" un essart, chacun travaille strictement sur ses futures parcelles, et c'est seul que, pendant des mois, un homme construit l'éventuelle barrière qui entoure son jardin. C'est presque exclusivement entre mari et femme que s'établit une coopération, l'un et l'autre effectuant alors des tâches complémentaires. Notons encore que, comme c'est souvent le cas, le degré d'entraide lors des travaux de jardinage se retrouve dans d'autres activités, par exemple lors de la construction des maisons individuelles : chez les Baruya, une vingtaine d'hommes et autant de femmes peuvent se rassembler pour édifier ensemble, et - insiste-t-on - dans la même journée, la demeure d'un jeune couple ; en revanche, il n'est pas rare de voir un Ankave construire pendant des semaines sa maison, toujours seul au travail, mais plaisantant à longueur de journée avec une demi-douzaine de parents ou d'amis venus lui rendre visite.

\section{Terre, nourriture ef échanges : une logique d'ensemble}

La forme que prennent localement les rapports entre affinité et coopération doit être rapprochée de cet autre trait variable des organisations sociales anga que sont les modalités du mariage. 
Les Anga du nord mettent l'accent sur l'échange direct de femmes alors que d'autres Anga accompagnent le mariage du versement d'une compensation matrimoniale, y compris lorsqu'il arrive que se réalise un échange de sœurs. Partout, les relations entre affins comportent une série continue de dons de gibier, qui débutent avec les "fiançailles", lorsque la future épouse est encore une fillette, pour ne s'achever qu'avec la mort de l'un des membres du couple. Mais c'est seulement là où existe un bridewealth (en monnaie de coquillages essentiellement) que l'on trouve de la viande de porc au premier rang des "biens" régulièrement offerts aux donneurs de femmes. On constate alors que la coopération lors du travail horticole est faible ou inexistante là où ces dons de porc sont importants.

Nous interpréterons cette corrélation quasi absolue de la manière suivante : lorsque le porc est de facto tenu pour un équivalent du corps de l'épouse, les affins ne peuvent participer aux travaux de défrichage, de protection ou d'entretien des jardins qui produisent les tubercules dont sont nourris les animaux reçus dans le cadre des relations d'alliance. Pour les Anga, l'idée ne serait pas tant que l'on ne peut manger ses propres porcs ${ }^{21}$, très répandue en Nouvelle-Guinée, que l'impossibilité de participer par son propre travail à la nourriture d'un animal qui vient "remplacer" une fille. Ce n'est là qu'une hypothèse, mais elle se trouve confortée lorsque l'on considère de près les cas qui pourraient a priori constituer des contre-exemples, puisqu' on y voit de la viande de porc circuler dans le cadre du mariage, alors même que celui-ci prend la forme d'un échange de sœurs et qu'il y a coopération entre affins.

Ainsi, le mariage baruya est précédé par un échange particulier - dit "construire le pont" (entre les deux familles) - qui se déroule en deux temps.
Lorsque les seins de la jeune fiancée se développent, les parents du garçon offrent un demiporc à la famille de celle-ci, qui en distribue aussitôt la majeure partie, constituée de morceaux gras. Plus tard, un autre demi-porc (l'autre moitié du "pont") est similairement donné aux parents du fiancé et transféré aux sœurs de sa mère. Ce don de viande est certes reçu par les parents paternels de la jeune fille (et, plus tard, du garçon), mais ceux-ci ne semblent pas le consommer - en tout cas, les mères des fiancés ne peuvent pas en manger. Il est au contraire transmis à des parents qui ne participent pas aux échanges de travail qui se sont instaurés dès que le mariage a été envisagé. Bref, parmi les dons réciproques de gibier (mais aussi, dans une moindre mesure, de porc) qui accompagnent le mariage baruya, celui que l'on cite spontanément comme un moment important du mariage lui-même prend la forme d'un paiement pour le corps d'un individu aux parents qui n'ont pas intégré celui-ci dans leur propre groupe, et les personnes qui consomment la viande ainsi offerte ne sont pas celles qui auraient pu produire les tubercules qui ont servi à nourrir l'animal dont elle provient.

Les Watchakes, un autre groupe anga du nord, présentent une situation du même type. Ils se marient traditionnellement par échange direct de femmes, et la coopération entre affins est la règle. Cependant, une fois marié, le jeune couple offre un porc entier aux parents de l'épouse, et la naissance d'un premier enfant s'accompagne du don d'un porc aux parents maternels de la femme qui vient d'accou-

21. Your own pigs you may not eat, pour reprendre le titre $d u$ célèbre ouvrage de P.G. Rubel et A. Rosman [1978]. 
cher. De même, lorsqu'un garçon subit les rituels d'initiation du premier stade (perforation du septum), ses parents donnent un porc à son oncle maternel. Ces dons prennent à nouveau la forme d'un dédommagement pour les enfants nés d'une sœur, mais, là encore, ceux qui les reçoivent n'ont généralement pas participé aux travaux dans les jardins d'où provient la nourriture de ces animaux. C'est très clairement le cas du porc reçu par les parents de l'épouse, dont on précise qu'il doit être acheté et non pas élevé par le couple lui-même, et de celui reçu par les maternels d'une jeune accouchée. Seul le frère de la mère qui reçoit un porc à chaque initiation d'un neveu utérin peut coopérer avec son beau-frère pour "produire" indirectement l'animal en question, au moins théoriquement.

Toujours au sein des Anga du nord, les Langimar offrent une dernière démonstration a contrario de l'hypothèse émise plus haut. Chez eux, la coopération est rare, mais chaque mariage - qui peut prendre la forme d'un échange de sœurs s'accompagne de dons aux parents de l'épouse, et, notamment, à plusieurs reprises, du don d'un plastron de porc. Cette fois, c'est seulement lorsque le mariage s'effectue sans échange de sœurs, ou bien lorsque le couple s'installe chez les parents de l'épouse parce que le mari ne dispose pas de suffisamment de terre chez ses propres parents, que s'instaure une coopération entre affins. Mais, dans les deux cas, celle-ci se fait à sens unique, si l'on peut dire, puisque, si l'époux travaille pour sa belle-famille, celle-ci ne vient pas l'aider à défricher, enclore ou planter le jardin du jeune gendre et beau-frère. Si bien que les Langimar établissent deux équivalences, l'une entre le travail et le corps de l'épouse, l'autre entre le travail et l'accès à la terre, qui sont dans le droit fil - à tout le moins dans le même registre - de l'hypothèse proposée. À nouveau, on ne coopère pas à la production d'un porc que l'on reçoit dans le cadre d'une relation d'alliance, et c'est le travail investi dans l'horticulture qui fonde une équivalence entre l'animal élevé sur une terre et le groupe de parents qui cultive le jardin et élève l'animal.

L'éventuelle présence de viande de porc parmi les prestations matrimoniales nous renseigne sur un lien fondamental entre la terre et l'organisation sociale chez les Anga. Mais il est une autre institution, et d'autres échanges, dans lesquels la "place" de l'animal nous interpelle, mais plus indirectement cette fois : il s'agit des initiations masculines, lors desquelles le porc n'est jamais consommé. Le gibier cérémoniel est constitué de marsupiaux, et lorsqu'il arrive que du porc soit offert aux initiés eux-mêmes, c'est soit avant les rituels, au moment de poser un interdit sur la consommation de cette viande, comme chez les Langimar [Blackwood 1978 : 127], soit lors du festin collectif qui marque la fin des rites proprement dits et la réinsertion des initiés dans l'espace profane villageois, comme chez les Baruya. Pendant les cérémonies elles-mêmes, le porc est strictement interdit aux initiés. Si l'on se rappelle que les initiations masculines séparent les jeunes garçons du monde féminin afin de les transformer en hommes et en guerriers sans l'intervention des femmes - même si c'est parfois à l'aide de substances d'origine féminine qu'il importe alors de "déféminiser" [Bonnemère, à paraître] -, il est probable que le porc ne peut y jouer aucun rôle car il évoque trop le monde féminin, du fait de l'équivalence de facto établie entre l'animal et le corps des femmes elles-mêmes (dans le mariage), ou de la part dominante que prennent celles-ci 
Une vallée baruya (ici Wonenara) se présente comme un damier de jardins en exploitation et de taches de forêt secondaire en train de repousser. Les fonds de vallées sont occupés par des jardins quasi permanents et par des champs de canne à sel. Les forêts qui constituent les territoires de chasse occupent le sommet des crêtes et les massifs montagneux inhabités qui séparent deux vallées.

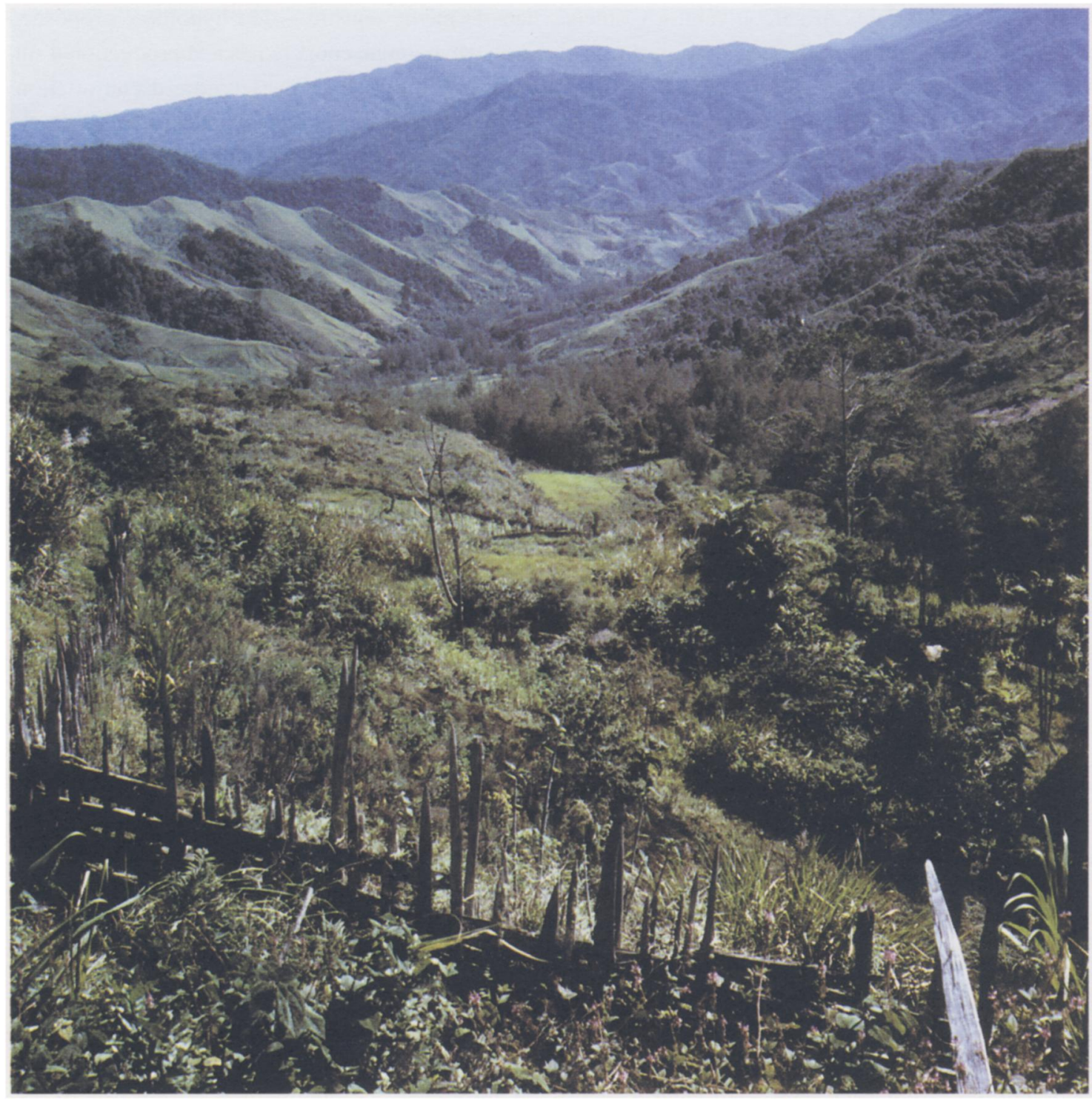

dans leur élevage et dans leur reproduction ${ }^{22}$. terre et le produit indirect de celle-ci. À l'inverse, Chez les Anga, les végétaux établissent une médiation entre le sol sur lequel ils croissent et un moyen d'échange, le porc. Notons que c'est à nouveau le travail qui établit ici un lien entre une

22. Ailleurs en Nouvelle-Guinée, lorsque l'animal est associé aux rituels masculins - comme sur le littoral sud de l'île - c'est de verrats sauvages qu'il s'agit, qui sont situés du côté du gibier et de la bravoure [Lemonnier 1993]. 


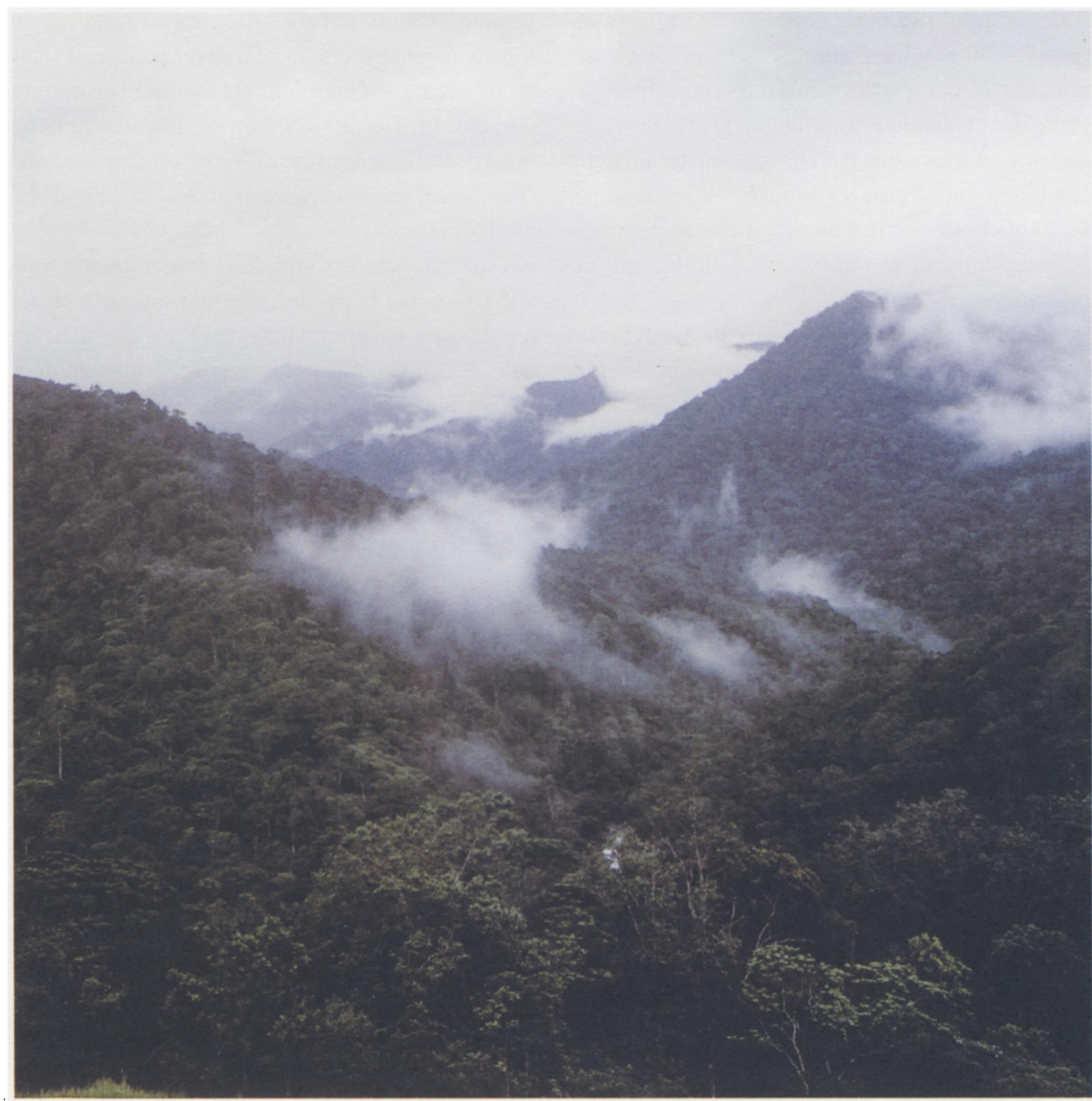

les marsupiaux évoquent la forêt, et, qu'ils soient tués à la flèche, piégés ou capturés à la main, c'est toujours par des hommes. De même, après une naissance, ils sont l'un des rares "objets" produits par un homme et seulement par lui. Si l'on excepte les anguilles, c'est même, pour un Anga, le seul qui traduise à la fois un lien avec une terre transmise en ligne patrilinéaire et une activité uniquement masculine. Ajoutons que sous ce rapport - celui qui s'établit entre un gibier et une terre -,
Par contraste, dans un paysage ankave, seul un œil très exercé peut deviner l'emplacement des jardins récemment abandonnés. Les hameaux euxmêmes se détachent à peine de la forêt omniprésente; quant aux campements provisoires, ils restent invisibles. 
il importe de distinguer entre les marsupiaux offerts par un homme à son épouse qui vient d'accoucher et celui offert collectivement dans le cadre des cérémonies d'initiation. Dans le premier cas, les animaux ont été chassés sur le territoire clanique (voire lignager) du mari, et, souvent, par lui seul. Lors des initiations, c'est au contraire l'aspect collectif et indifférencié du don qui est mis en avant.

Il est une circonstance au cours de laquelle les Ankave illustrent ces deux origines - et destinations - possibles du gibier. Lors d'une première naissance (en l'occurrence lorsqu'un homme a un premier enfant), deux chasses se succèdent. D'abord, plusieurs hommes aident le jeune père à capturer des marsupiaux sur les territoires claniques de ceux qui les ouvrent à autrui pour l'occasion. Au retour d'une cêrémonie en forêt qui marque l'accession du jeune père au troisième et dernier stade initiatique, ce gibier est offert aux parents maternels du couple, mais aussi à toutes les femmes présentes, y compris celle qui vient d'accoucher. Quelques jours plus tard, le nouveau père repart chasser, mais cette fois le plus souvent seul et uniquement dans la portion de forêt qui lui revient. Alors que les premiers dons, collectifs et sans référence à un territoire particulier, constituent en quelque sorte le dernier acte de la cérémonie d'initiation du père et concernent les femmes en général, les seconds sont, strictement, un don du mari à son épouse.

Dans deux autres groupes anga au moins (les Menye et les Watchakes), le gibier offert aussitôt après une première naissance diffère de la même manière de celui qu'un mari donne à son épouse à l'occasion de toute naissance. Dans tous les cas, il importe que ce dernier gibier, offert à une femme au moment où elle vient de mettre au monde un enfant qui va se trouver incorporé dans le groupe de son mari, ait été capturé sur la terre de celui-ci. La faible mobilité des marsupiaux, qui vivent dans des trous d'arbre ou dans des terriers, accentue encore ce lien avec un territoire particulier.

À l'inverse, lors des initiations masculines, même s'il arrive que la capture des marsupiaux s'effectue dans un territoire clanique plutôt que dans un autre - et notamment dans les forêts du clan dont sont originaires les maîtres des initiations qui officient, ou dans celle de la communauté locale où se déroule la cérémonie -, tous les hommes y participent. Ils se rassemblent pour exploiter un même territoire ou, lorsqu'ils chassent les marsupiaux dans leurs propres brousses, pour constituer un même stock de gibier. À chaque fois, le gibier reçu par un initié est anonyme.

\section{Substances corporelles et marqueurs du territoire}

Comme on le voit, les conditions dans lesquelles s'établit l'association d'une activité productrice et d'une référence à un territoire déterminent dans une large mesure le statut symbolique des biens d'échange chez les Anga. Pour cette raison, la matérialisation des limites entre les espaces appropriés par les clans, les lignages ou les individus est de toute première importance et conduit à s'interroger sur ce que ces sociétés inscrivent dans les marqueurs de l'espace au sein duquel on jardine ou on chasse.

De même que, dans un jardin, les parcelles exploitées par plusieurs femmes sont toujours délimitées d'une manière ou d'une autre (arbres caractéristiques, troncs posés sur le sol, alignements de 
fleurs), on trouve des marqueurs à l'intérieur du territoire sur lequel peuvent venir chasser et cultiver tous les hommes d'un clan. Ce sont le plus souvent des arbres - pandanus ou palmiers-aréquiers - et parfois des nids de volaille sauvage qui constituent les bornes délimitant l'espace horticole d'un lignage donné lorsque la forêt secondaire a repoussé, entre deux cycles de défrichage et de jardinage. Le Pandanus julianetti ne pousse qu'en altitude et ne sert donc généralement de marqueur que dans les brousses de chasse situées au-dessus de 2100 mètres, mais le Pandanus conoideus ou l'Areca catechu sont également plantés dans les jardins. Leurs fruits sont très appréciés et leur consommation est toujours un important moment de sociabilité : on offre des noix d'arec à ceux que l'on rencontre en chemin ; quant à la dégustation du jus de pandanus rouge, elle prend souvent une allure cérémonielle.

Au moment de l'ouverture des jardins elle-même, certains groupes mettent davantage d'emphase que d'autres dans leur délimitation. On a vu que chez les Baruya, par exemple, en même temps qu'elles sont un moyen d'empêcher les porcs de détruire les cultures et de délimiter le sol qu'un homme a mis à la disposition de diverses familles, les barrières matérialisent des relations cruciales entre les affins ou les co-initiés qui ont intensément collaboré pour les construire. Plus généralement, lorsqu' on se déplace dans la forêt, à tout moment un Anga indique spontanément les noms de ceux dont on voit les arbres. Dans tous les groupes anga, les marqueurs qui indiquent les limites des territoires de chacun sont des témoins choisis pour leur stabilité dans le temps. Cela est évident pour les arbres qui, une fois qu'ils ont "pris", par rejet naturel ou après avoir été replan- tés, restent fixés au sol. Mais cela est aussi le cas des monticules des mégapodes car, comme le disent les Watchakes, l'oiseau revient toujours déposer ses œufs au même endroit ${ }^{23}$. Mais il s'agit aussi de ressources sauvages domestiquées, ou tout au moins contrôlées, par un travail masculin (et non par les femmes). Ces arbres n'ont pas seulement été repérés ou plantés par leur premier utilisateur ; il faut aussi les entretenir régulièrement, nettoyer le sol à leur pied et dégager les arbres alentour.

Il est à remarquer que le pandanus et l'aréquier ne présentent en eux-mêmes aucune rareté ; n'importe qui peut en planter et en offrir les fruits. Voler ces derniers, ce n'est pas tant se procurer un bien convoité qu'attenter aux droits d'autrui sur son sol. Par exemple, c'est au moment où un jardin cesse de produire qu'un Ankave plante un Pandanus conoideus. Comme un pandanus ne porte pas de fruits avant quatre ans pleins, c'est bien le rapport entre un sol, une nourriture et un lignage que l'on veut rappeler puis matérialiser dans le temps. Pour un homme, déclarer que son père ou son grand-père a planté un aréquier ou un pandanus à un endroit donné revient à affirmer un droit d'usage exclusif sur la terre environnante. Inversement, lors de 1'enquête sur les conditions dans lesquelles on peut accueillir un parent ou un ami sur son sol, des informateurs précisent qu'il s'agit pour les invités de planter "seulement de la nourriture" - patate douce, taro, canne à sucre, bananier, etc. - et jamais aucun de ces arbres qui indiquent un droit sur la terre. A contrario, lorsqu'un Ankave cède

23. À l'inverse, pour certains groupes, tous les oiseaux peuvent être chassés par n'importe qui, car "ils passent de brousse en brousse". 


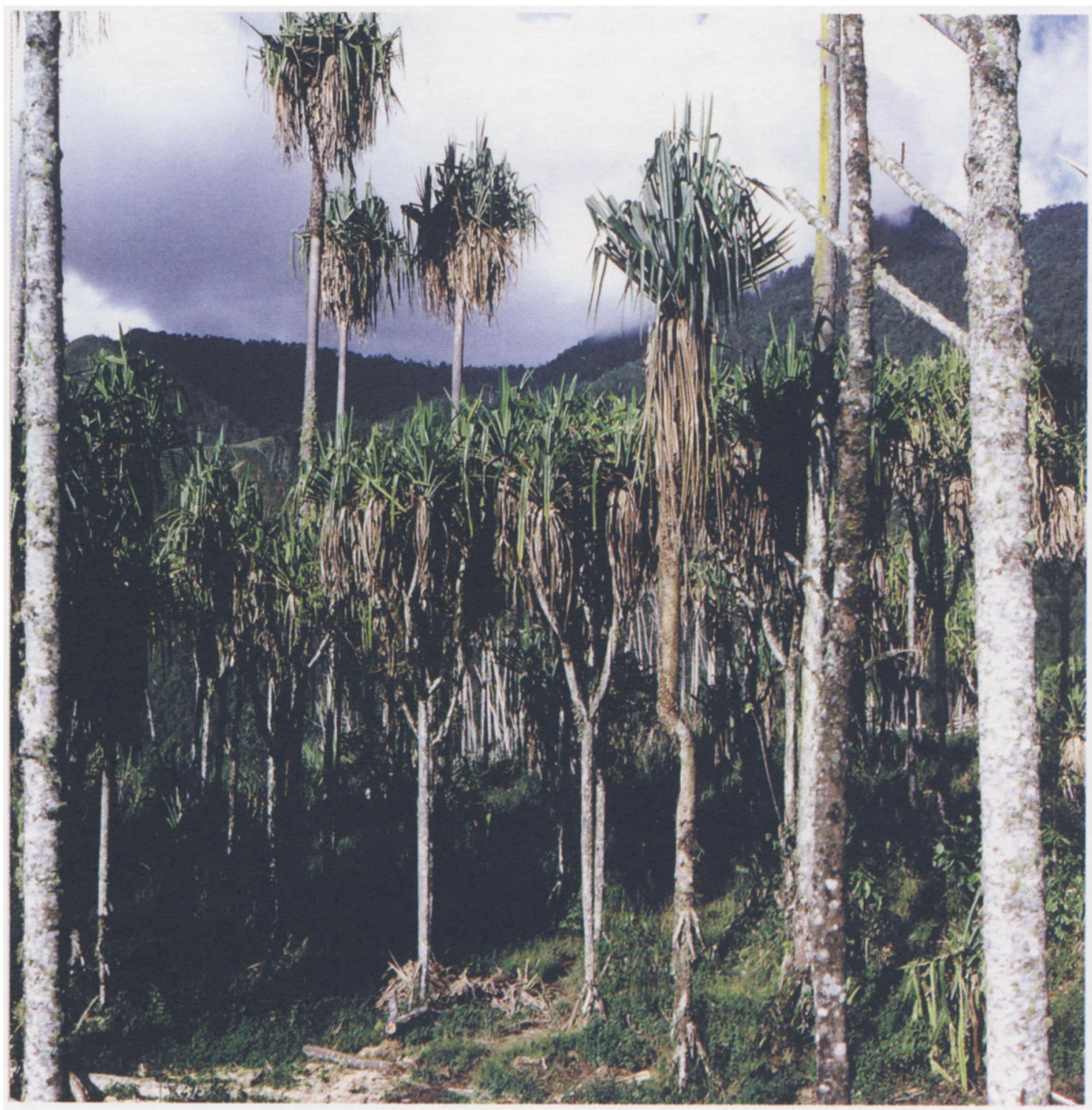

définitivement de la terre à un homme qui n'est pas de son clan, il ne dit pas qu'il lui a donné un sol, mais un pandanus rouge.

Le choix même de ces arbres qui servent de mar- queurs n'est pas indifférent. Il dépend évidemment des espèces disponibles sur le territoire d'un groupe donné, mais, parmi celles-ci, certaines seulement sont culturellement investies des qualités qui 


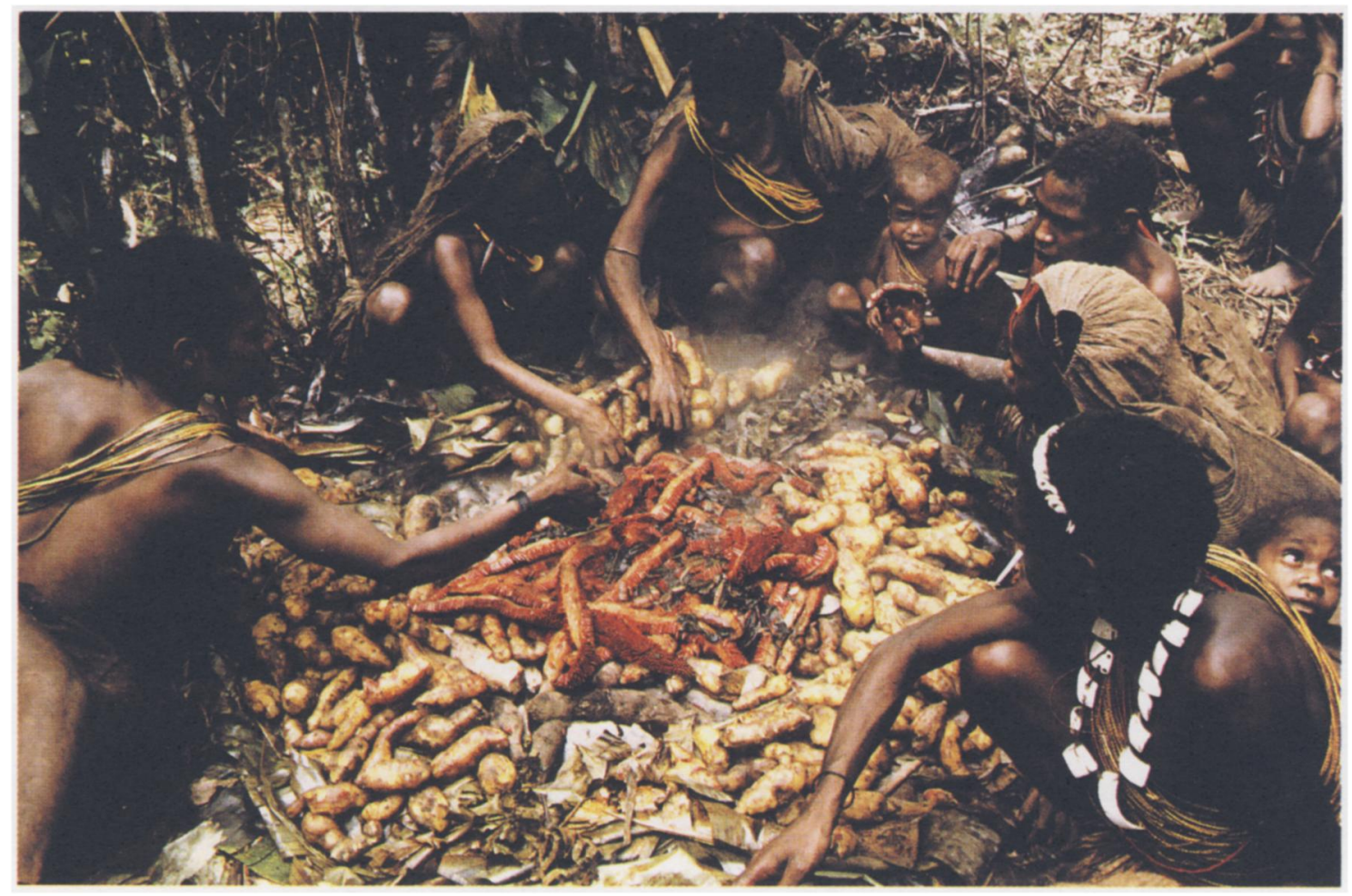

Pour les Anga du nord, les fruits du $P$. julianetti produisent du sperme dans le corps des hommes; pour les Ankove, en revanche, c'est au sang fóminin qu'est explicitement associé le jus rouge extrait des graines de P. conoideus (ici en cours de cuisson).

en font le symbole d'un droit sur la terre. Comme on l'a vu, l'aréquier est partout l'un de ces marqueurs. Les pandanus en sont un autre, mais, alors que les Anga du nord conferent une grande importance aux Pandanus julianetti, qui forment des bosquets bien entretenus aux pieds desquels pousse souvent un tapis de fleurs décoratives, c'est dans le Pandanus conoideus que les Ankave voient le prototype du marqueur de territoire. Or, les rôles de ces arbres ne se limitent pas là. Pour les Anga du nord, le Pandanus julianetti est associé au sperme, et notamment à sa régénération. Les Sambia ingèrent la sève blanchâtre qui s'écoule de leurs racines aériennes pour remplacer la substance séminale qu'ils ont perdue lorsque les initiés s'en sont nourris par fellation [Herdt 1981 : 111]. Pour les Baruya, c'est en consommant le fruit de ce même pandanus que la femme primordiale devint enceinte [Godelier 1982 : 243]. Pour leur part, les Ankave tiennent le Pandanus julianetti pour un arbre ordinaire, et c'est d'abord à d'autres végétaux, ainsi qu'à certaines larves, qu'ils attribuent le pouvoir de fabriquer du sperme dans le corps des hommes. En revanche, ils considèrent que le jus rouge vermillon extrait des fruits de l'autre pandanus (Pandanus conoideus) est l'équivalent du sang menstruel, et l'un des moments cruciaux des initiations masculines est l'onction des graines cuites de ces fruits sur le corps des jeunes garçons, qui acquièrent ainsi leur 
maturité et leur force d'homme. Notons que les initiations ankave mettent en scène une autre relation territoriale dont le sang est l'un des vecteurs : la terre utilisée pour enduire le corps des initiés est censée toujours contenir en elle le sang d'un ancêtre tué tantôt sur le site d'origine de tous les Anga, tantôt à la frontière des deux principales vallées de la tribu, selon la version du mythe. Là où le corps de cet homme fut exposé, puis mangé, ont poussé les cordylines rouges qui, génération après génération, servent à délimiter les enceintes sacrées où se déroulent les initiations. À la fin de celles-ci, chaque homme adulte en replante un pied parmi les cordylines "ordinaires" (vertes) qui entourent son enclos domestique. Lors des rites suivants, il le recoupe pour constituer l'aire cérémonielle.

Le contraste est donc grand entre les initiations masculines des Anga du nord, qui mettent l'accent sur le rôle du sperme et de ses substituts et comportent des pratiques d'homosexualité ritualisée, et celles des Ankave, qui font une large place au sang et sont dépourvues de pratiques homosexuelles. Mais, dans l'un et l'autre cas, la manière dont on fait renaître les garçons dans un monde masculin entretient une étroite relation d'homologie avec les théories locales de la personne et de la procréation. Pour les Anga du nord, en effet, le sperme est seul responsable de la constitution de l'embryon puis du développement du fœtus. Par la suite, le soleil intervient pour achever le nouvel être humain, mais la mère n'a aucun rôle actif dans le processus de fabrication d'un être, sa matrice n'étant qu'un 'contenant" [Godelier 1982 : 90-91]. À l'inverse, c'est au sang maternel (et menstruel, qui lui est identifié) que les Ankave attribuent l'essentiel de la croissance de l'embryon, dans la constitution initiale duquel les principes masculins et féminins interviennent à part égale [Bonnemère 1993a : 368 sq.].

En d'autres termes, les arbres avec lesquels les Anga délimitent leurs territoires claniques sont aussi ceux dont la collectivité des hommes tire les équivalents des substances corporelles avec lesquelles les jeunes garçons sont transformés en guerriers adultes. Un même ensemble de représentations sous-tend donc à la fois les théories de la personne, la structure des initiations masculines et le marquage des territoires.

Notons pour finir que de la terre elle-même, en tant qu'elle est une substance, les Anga parlent peu. En particulier, ils restent muets sur ce que nous appellerions sa fonction de support de la vie végétale. Certes, ils effectuent de multiples pratiques magiques pour assurer le succès des récoltes [Bonnemère 1993a : 273-274 ; Godelier 1973 : 358-361], mais sans préciser la façon dont celles-ci opèrent. De même, en l'absence de discours sur ce thème, c'est seulement par analogie avec ce que l'on sait des pratiques d'autres régions de Nouvelle-Guinée que l'on peut discerner un intérêt pour le "recyclage" de certaines substances corporelles à la mort des individus. Hormis l'usage de la terre imprégnée du sang de l'ancêtre ankave, les seuls cas où la terre elle-même est investie de quelque pouvoir sont les onctions de diverses terres destinées à refroidir le corps, à en écarter la maladie ou à le vivifier, mais, le plus souvent, l'origine de ces pouvoirs n'est pas commentée [Bonnemère 1993a : 415-419; Ollier et al. 1971 : 34-36]. 
mêmes, participent de manière fondamentale à la circulation d'un élément du corps - sperme ou

Finalement, c'est surtout de trois manières que les Anga établissent des relations entre des conceptions relatives à la terre et leur organisation sociale. Premièrement, tous reconnaissent implicitement que le travail investi dans la production des tubercules dont on nourrit un porc est constitutif de la valeur symbolique attribuée à un animal particulier. Les variations concomitantes de l'intensité de la coopération entre affins et de la place faite à la viande de porc dans les échanges liés au mariage apparaissent comme une conséquence de cette relation. Ensuite, la destination du gibier n'est pas la même selon qu'il provient d'une ou de plusieurs brousses de chasse. Celui qui est capturé sur un territoire clanique ou lignager particulier est offert par un homme à ses affins ou à son épouse ; celui qui provient de plusieurs territoires à la fois est utilisé dans le cadre de ces événements collectifs que sont les initiations. Notons que la référence à la localité et celle au travail sont toujours imbriquées dans la définition d'un produit - porc ou gibier - distinguable de celui d'un autre groupe ou individu. Dans le cas des jardins, le travail d'un premier défricheur octroie des droits d'usage sur un sol particulier, mais c'est aussi par leur travail que ceux qui y ouvrent et cultivent un jardin inscrivent leur identité dans les produits directs et indirects (porcs) de celui-ci ${ }^{24}$. De même, c'est parce qu'un ancêtre y a posé des pièges ou entretenu des arbres le premier que ses descendants disposent d'un droit d'usage exclusif sur une portion de forêt [Godelier 1969 : 4].

Enfin, le rôle discriminant conféré à la localité implique des procédures de délimitation de l'espace. Celles-ci font appel à des marqueurs qui, en eux- sang - par lequel les Anga établissent deux ensembles de relations fondamentales : dans le domaine de la parenté, celles qui, à travers un partage de substances, unissent plus particulièrement un individu à ses parents paternels ou maternels ; et lors des initiations, celles qui sous-tendent le processus de transformation des garçons en hommes accomplis, voire chez les Ankave la relation à une histoire et à un ancêtre communs, qui est constitutive de la collectivité des hommes, par opposition à celle des femmes.

Chez les Anga comme souvent ailleurs en Nouvelle-Guinée, il est difficile de prédire selon quelles lignes diverses unités sociales se clivent en cas de tension. Par exemple, lorsqu'un clan baruya intervient dans un conflit qui oppose deux autres clans, ses membres se répartissent de manière à peu près égale auprès des deux partis en présence (Godelier : communication personnelle). De même, on ne sait pas toujours quels principes permettraient de rendre compte à tout coup de la composition d'un groupe local. Dans un village baruya, on trouve souvent des représentants masculins de 6 ou 7 clans différents, dont les deux plus nombreux rassemblent 50 ou $60 \%$ des résidents $^{25}$; les terres cultivables les plus proches sont celles du clan majoritaire, mais la moitié des femmes qui y exploitent des parcelles le font sans aucun droit de "propriété", seulement au titre de l'alliance ou de l'entraide [Godelier $1969: 21$ ]. La

\footnotetext{
24. D. de Coppet a noté une semblable "exposition" de l'effort et de la fatigue dans les jardins are'-are' [1976: 169-170].

25. Ces chiffres ont été établis à partir des recensements de population que M. Godelier a bien voulu nous communiquer.
} 
situation est globalement semblable chez les Ankave : il existe certes des hameaux où plus de $75 \%$ des hommes sont membres du clan possédant les terres attenantes, mais il y en a d'autres où les non-agnats sont majoritaires, notamment lorsque leurs propres terres se trouvent à faible distance.

Dans les deux groupes cependant, les hommes résidant au sein d'un hameau qui n'est pas situé sur leurs terres claniques sont essentiellement - et même presque exclusivement pour les Ankave ${ }^{26}$ des affins des membres du clan local. C'est dire l'importance du mariage dans la constitution des communautés territoriales. On doit aussi noter que, parallèlement à leur rôle dans la reproduction des rapports entre les sexes, pour les Anga, les initiations masculines font l'unité de la tribu, en tant que tous ses membres masculins exploitent et défendent globalement un même territoire. Si bien que le mariage et les initiations apparaissent étroitement liés à la définition de deux importantes entités simultanément sociologiques et territoriales : le village et la tribu. Une référence à la terre est en filigrane dans les relations entre les membres de ces deux types de communautés, mais elle est le plus souvent indirecte et doit être construite à travers le partage ou l'échange de nourritures et de substances. On a montré ici que, dans leur contenu symbolique même, ces biens, dont l'échange est au fondement du mariage ou des initiations, se définissent par une référence à la terre dont le travail est indissociable.

26. Dans les deux hameaux les plus peuplés de la vallée de la Suowi, les affins du clan local représentent 29 et $46 \%$ du total des hommes adultes.

\section{Références bibliographiques}

\begin{abstract}
Allen, M.R. - 1984, "Homosexuality, male power, and political organization in North Vanuatu : a comparative analysis", in G. Herdt, ed., Ritualized homosexuality in Melanesia. Berkeley, Los Angeles, Londres, University of California Press : 83-126.

Barnes, J.A. - 1962, "African models in the New Guinea Highlands", Man 62 (2) : 5-9.

Barrau, J. - 1962, "Les plantes alimentaires de l'Océanie. Origines, distribution et usages", in Annales du Musée colonial de Marseille. Marseille, Musée colonial de Marseille. 1972, "Culture itinérante, culture sur brûlis, écobuage ou essartage : un problème de terminologie agraire", Etudes rurales $45:$ : 99-103.

Blackwood, B. - 1950, The technology of a modern Stone Age people in New Guinea. Oxford, Pitt Rivers Museum. 1978, The Kukukuku of the Upper Watut. Oxford, Pitt Rivers Museum, University of Oxford.
\end{abstract}

Bonnemère, P. - 1993a, Le casoar, le pandanus rouge et l'anguille. Différence des sexes, substances et parenté chez les Ankave-Anga de Papouasie Nouvelle-Guinée. Thèse de doctorat. Paris, EHESS. - 1993b, "Pangium edule : a food for the social body among the Ankave-Anga of Papua New Guinea", in C.-M. Hladik et al., eds., Tropical forests, people and food : biocultural interactions and applications to development. Paris, UNESCO/Parthenon Publishing Group. "Echanges et traitement du corps : les représentations de la croissance et de la maturation chez les Ankave-Anga (Papouasie Nouvelle-Guinée)", in M. Godelier et M. Panoff, eds. Le corps humain : expression et instrument des ordres et des désordres dans la société et le cosmos. Paris, à paraître.

Brown, P. - 1962, "Non-agnates among the patrilineal Chimbu", Journal of the Polynesian Society 71 : 57-64.

Coppet, D. de - 1976, "Jardins de vie, jardins de mort en Mélanésie", Traverses 4-6 : 60-70. 
Feil, D.K. - 1987, The evolution of Highland Papua New Guinea societies. Cambridge, Cambridge University Press.

Fischer, H. - 1968, Negwa. Eine Papua Gruppe im Wandel. Munich, Klaus Renner Verlag.

Frankel, S. - 1986, The Huli response to illness. Cambridge, Cambridge University Press.

Gillison, G. - 1983, "Cannibalism among women in the Eastern Highlands of Papua New Guinea", in P. Brown et D. Tuzin, eds., The ethnography of cannibalism. Washington, The Society for Psychological Anthropology : 33-50.

Glasse, R.M. - 1968, Huli of Papua. A cognatic descent system. Paris, La Hague, Mouton.

Godelier, M. - 1969, "Land Tenure among the Baruya of New Guinea", Journal of the Papua New Guinea Society 3 : 17-23. - 1973, Horizon, trajets marxistes en anthropologie. Paris, Maspero : 258-270. - 1982, La production des Grands hommes. Pouvoir et domination masculine chez les Baruya de Nouvelle-Guinée. Paris, Fayard.

Godelier, M. et J. Garanger - 1973, "Outils de pierre, outils d'acier chez les Baruya de Nouvelle-Guinée. Quelques données ethnographiques et quantitatives", L'Homme 13 : 186-220.

Heider, K.G. - 1970, The Dugum Dani. A Papuan culture in the Highlands of West New Guinea. New York, WennerGren Foundation for Anthropological Research (Viking FuniPublication in Anthropology 49).

Herdt, G. - 1981, Guardians of the flute. Idioms of masculinity. New York, McGraw-Hill. - 1987, The Sambia. Ritual and gender in New Guinea. New York, Holt, Rinehart and Winston.

Iteanu, A. - 1983, La ronde des échanges. De la production aux valeurs chez les Orokaiva. Cambridge, Paris, Cambridge University Press/Éd. de la MSH.

Kahn, M. - 1986, Always hungry, never greedy. Food and the expression of gender in a Melanesian society. Cambridge, Cambridge University Press.

La Fontaine, J. - 1973, "Descent in New Guinea. An africanist view", in J. Goody, ed., The Character of Kinship Cambridge, Cambridge University Press : 35-51.

Landiman, G. - 1927, The Kiwai Papuans of British Nev $\rightarrow$ Guinea. Londres, Macmillan.

$\rightarrow$ Langness, L.L. - 1964, "Some problems in conceptualisation of Highlands social structure", American Anthropologist 66 (4) : 162-182. - 1972, "Political organization", in P. Ryan, ed., Encyclopædia of Papua and New Guinea. Melbourne, Melbourne University Press/University of Papua and New Guinea : 924-935.

Lawrence, P. - 1984, The Garia. An ethnography of a traditional cosmic system in Papua New Guinea. Manchester, Manchester University Press.
Lemonnier, P. - 1981, "Le commerce inter-tribal des Anga de Nouvelle-Guinée", Journal de la Société des Océanistes 37 (70-71) : 39-75. - 1982, "Les jardins anga (NouvelleGuinée)", Journal d'Agriculture tropicale et de Botanique appliquée 29 (3-4) : 226-245. - 1990, Guerres et festins. Paix, échanges et compétition dans les Highlands de Nouvelle-Guinée. Paris, Éd. de la MSH. - 1993, "Le porc comme substitut de vie : formes de compensation et échanges en Nouvelle-Guinée", Social Anthropology 1 (1A) : 33-55.

Lepervanche, M. de - 1967-1968, "Descent, residence and leadership in the New Guinea Highlands", Oceania 38 : 134-158 et 163-189.

LiPuma, E. - 1988, The gift of kinship. Structure and practice in Maring social organization. Cambridge, Cambridge University Press.

Lory, J.-L. - 1982, "Les jardins baruya", Journal d'Agriculture tropicale et de Botanique appliquée 29 (3-4) : 247 274.

Meggitt, M.J. - 1965, The lineage system of the Mae-Enga of New Guinea. Édimbourg, Londres, Oliver and Boyd.

Ollier, C.D., D.P. Drover, M. Godelier - 1971, "Soil knowledge among the Baruya of Wonenara, New Guinea", Oceania 42 (1) : 34-41

Pouwer, J. - 1964, "A social system in the Star Mountains. Toward a reorientation of the study of social systems", American Anthropologist 66 (4) : 133-161.

Rappaport, R.A. - 1968, Pigs for the ancestors. Ritual in the ecology of a New Guinea people. New Haven, Londres, Yale University Press.

Rubel, P.G., A. Rosman - 1978, Your own pigs you may not eat. A comparative study of New Guinea societies. Canberra, Australian University Press.

Ryan, D.J. - 1959, "Clan formation in the Mendi valley", Oceania 29 : 257-289.

$\rightarrow$ Salisbury, R.F. - 1964, "New Guinea Highlands models and descent theory", Man 64 : 168-171.

Scheffler, H.W. - 1985, "Filiation and affiliation", Man 20 (1): 1-21.

Sillitoe, P. - 1978, "Big men and war in New Guinea", Man 13 (2) : 252-271. - 1979, Give and take. Exchange in Wola society. Canberra, Londres, Australian National University Press. - 1983, Roots of the earth. Crops in the Highlands of Papua New Guinea. Kensington, New South Wales University Press.

Strathern, A.J. - 1969, "Descent and alliance in the New Guinea Highlands : some problems of comparison", Proceedings of the Royal Anthropological Institute of Great-Britain and Ireland for 1968 : 37-52. - 1973, "Kinship, descent and locality : some New Guinea examples", in J. Goody, 
ed., The character of kinship. Cambridge, Cambridge University Press. - 1979, " 'It's his affair'. A note on the individual and the group in New Guinea Highlands societies", Canberra Anthropology 2 (1): 98-113. - 1981, "Death as exchange : two Melanesian cases", in S.C. Humphries et H. King, eds., Mortality and immortality. The anthropology and archaeology of death. Londres, Academic Press. - 1982a, "Two waves of African models in the New Guinea Highlands", in A.J. Strathern, ed., Inequality in the New Guinea Highlands societies. Cambridge, Cambridge University Press : 35-49. - 1982b, "Witchcraft, greed, cannibalism, and death. Some related themes from the New Guinea Highlands", in M. Bloch et J. Parry, eds., Death and the regeneration of life. New York, Pergamon : 111-133.

Strathern, M. - 1972, Women in between. Female roles in a male world: Mount Hagen, New Guinea. New York, Londres, Seminar $\mathrm{Pr} \rightarrow-1984$, "Marriage exchanges. A Melanesian comment", Annual Review of Anthropology 13 : 41-73. - 1988, The gender of the gift. Problems with women and problems with society in Melanesia. Berkeley, Los Angeles, Londres, University of California Press.

van Baal, J. - 1966, Dema. Description and analysis of Marindanim culture (South New Guinea). La Hague, Martinus Niphoff.

Wagner, R. - 1974, "Are there social groups in the New Guinea Highlands?", in J. Leaf, ed., Frontiers of anthropology. New York, D. van Nostrand Company : 95-122.

Weiner, J.F. - 1982, "Substance, siblingship and exchange. Aspects of social structure in New Guinea", Social Analysis $11:$ :3-34. 